As a result, the airline is facing an $£ 18$ billion class-action lawsuit filed in the High Court in London on behalf of customers impacted by the disclosures. The lawsuit refers to aspects of the General Data Protection Regulations (GDPR). This allows consumers the right to claim compensation when their information is compromised in security incidents. There could also be heavy fines for Easy Jet under GDPR.

\section{Losing control}

The most sophisticated publicised instance of hacking thus far remains Stuxnet. This complex attack on an Iranian nuclear facility exploited supervisory control and data acquisition (SCADA) systems. It was the first to demonstrate the extreme vulnerability of industrial control systems being connected. Since then, there have been numerous similar exploits, often employing parts of the Stuxnet code, since it became public.

In June this year, Honda's corporate network was breached. According to the security firm, Sentinel One, it found Ekans or Snake ransomware. Snake is designed to attack industrial control systems. The fact that Honda halted production in the UK, US, Turkey, Italy and Japan, implies some success.

Honda insisted that no data had been lost, suggesting they were able to restore from backups. This may have caught the problem early enough to minimise damage. There is no evidence yet of how the attackers gained initial access, but it is likely to have been part of a Covid-19 related phishing campaign.

\section{Celebrity scam}

Twitter was again the subject of a headlinegrabbing breach in early July, which saw high profile accounts like those of Joe Biden, Barack Obama, Elon Musk, Bill Gates, Jeff Bezos and Apple taken over by hackers. Subsequent tweets promised their legions of followers 'double their money' if they deposited bitcoins to a particular account. Despite the unlikely sounding offer, more than $\$ 100,000$ was actually deposited in the rogue Bitcoin account.

Such was the relatively amateur nature of this attack, authorities were able to identify and arrest the perpetrators within days. The 17-year-old 'mastermind' was also prepared to discuss details of the hack with journalists online. This attack was achieved through social engineering a number of Twitter employees over the phone.

\section{Navigating a hostile network}

In late July, users of Garmin satellite navigation devices and services found they were suddenly unable to use them.

The company's website was down, its call centre offline and, for a time, there was no way to contact them at all.

They had been attacked with WastedLocker, ransomware software developed by the notorious Russia-based Evil Corp group. Such was the disruption caused by the attack, that production was halted at Garmin's factories in Taiwan for five days.

\section{Chinese warning}

Companies operating in China were placed on alert late in the summer, after hidden backdoors were discovered in mandatory tax-related software. China's banks require historical interest in these sectors.

\section{The new normal}

As we come to terms with how the world may be changed as a result of Covid-19, there are indications the cybersecurity landscape is also changing.

From this brief selection of some of 2020's security incidents, it should be clear that they naturally fall into categories. There are tabloid-friendly hacks, like the one at Twitter - newsworthy only because of the names of those involved.

Then, there are the inevitable and escalating attacks on financial institutions and medical facilities with the growing use of ransomware as an effective means to extort money.

There are also the huge number of database breaches resulting in the theft of personal data. This problem could be said to have reached epidemic proportions on its own, as websites which try to catalogue all cyber incidents show data leaks on an almost daily basis.

Cyber criminals are industrialising their projects and state actors are being discovered taking even greater interest in

\section{'This complex attack on an Iranian nuclear facility exploited supervisory control and data acquisition (SCADA) systems.'}

all companies to download software from either Aisino or Baiwang to comply with its Golden Tax VAT scheme. This could indicate that the malware, labelled GoldenSpy, has either direct sponsorship from the government or is being deployed with its blessing.

More malware, dubbed GoldenHelper, which pre-dates GoldenSpy, has been found in the Golden Tax Invoicing Software (Baiwang edition) which is digitally signed by a subsidiary of Aisino, Nou Nou Technologies. GoldenHelper, while functionally different to GoldenSpy, has a similar delivery mechanism, according to security company, Trustwave.

Based on this, the FBI sent out an official warning to all US companies in China. They believed companies in the healthcare, chemical and finance sectors are in particular danger, based on China's large companies, while the ever-present threat to individuals is only getting more sophisticated. There is already evidence emerging of renewed interference by Russia in the 2020 US Presidential election. In a year when a real virus monopolised our attention, those in the digital realm continued to develop and mutate.

While there is the possibility of a vaccine for one contagion, to eradicate the other may be considerably more difficult.

\section{About the author}

Patrick O'Connor

(linkedin.com/in/pdoconnor)

is a principal systems analyst

programmer with Amadeus IT

Group (www.amadeus.com), the leading travel technology company. 